Genome Insight

Genomics and Bioinformatics

\title{
Comparative and phylogenetic analyses of Swertia L. (Gentianaceae) medicinal plants (from Qinghai, China) based on complete chloroplast genomes
}

\author{
Xin $\mathrm{Xu}^{1 *}$, Jinping $\mathrm{Li}^{2,3^{*}}$, Ran $\mathrm{Chu}^{1}$, Mengjie Luan ${ }^{1}$, Hongyu Wang ${ }^{2}$, Kexin Song ${ }^{1}$, Shixia Wei ${ }^{1}$, Yuhua Shi ${ }^{1}$, \\ Shixin Zhu ${ }^{1}$ and Zhen Wei ${ }^{1}$ \\ 'Zhengzhou University, School of Life Sciences, Zhengzhou, Henan, China. \\ ${ }^{2}$ Qinghai Normal University, School of Life Sciences, Xining, Qinghai, China. \\ ${ }^{3}$ Key Laboratory of Medicinal Animal and Plant Resources of Qinghai-Tibetan Plateau in Qinghai Province, \\ Xining, Qinghai, China.
}

\begin{abstract}
Swertia L. is a large genus in Swertiinae (Gentianaceae). In China, many Swertia species are used as traditional Tibetan medicines, known as "Zangyinchen" or "Dida". However, the phylogenetic relationships among Swertia medicinal plants and their wild relatives have remained unclear. In this study, we sequenced and assembled 16 complete chloroplast (cp) genomes of 10 Swertia species, mainly distributed in Qinghai Province, China. The results showed that these species have typical structures and characteristics of plant cp genomes. The sizes of Swertia cp genomes are ranging from 149,488 bp to $154,097 \mathrm{bp}$. Most Swertia cp genomes presented 134 genes, including 85 protein coding genes, eight rRNA genes, 37 tRNA genes, and four pseudogenes. Furthermore, the GC contents and boundaries of $\mathrm{cp}$ genomes are similar among Swertia species. The phylogenetic analyses indicated that Swertia is a complex polyphyletic group. In addition, positive selection was found in $p s a l$ and petL genes, indicating the possible adaptation of Qinghai Swertia species to the light environment of the Qinghai-Tibet plateau. These new cp genome data could be further investigated to develop DNA barcodes for Swertia medicinal plants and for additional systematic studies of Swertia and Swertiinae species.
\end{abstract}

Keywords: Swertia, Zangyinchen, chloroplast genome, Gentianaceae, Qinghai-Tibet Plateau.

Received: March 30, 2021; Accepted: October 03, 2021.

Swertia L., a large genus of plants in Swertiinae (Gentianaceae) with about 170 species, are mainly distributed in Asia, Africa, North America and Europe (Ho et al., 1994). In China, around 79 Swertia species are mostly distributed in mountainous areas of the southwest, especially in the Himalayas (Ho and James, 1995; Joshi and Joshi, 2008). Some Swertia species are often used as Tibetan medicines, known as 'Zangyinchen' or 'Dida' to treat hepatobiliary diseases (Zhong et al., 2009). Although Swertia species have a long history of medicinal use, the systematics of the genus and regional groups remain obscure (von Hagen and Kadereit, 2001, 2002; Kadereit and von Hagen, 2003; Xi et al., 2014). In the present study, we assembled and annotated the complete chloroplast (cp) genomes of 16 Swertia taxa (10 Swertia species) mainly distributed in Qinghai province, China. The chloroplast genomes of these Swertia species were compared and used to study the phylogenetic relationships and selection pressure.

Fresh leaves of the plants were collected mainly in the Qinghai area (Table S1). Total genomic DNA was extracted using a modified CTAB method (Wei et al., 2017) and then purified with a Wizard ${ }^{\circledR}$ DNA Clean-Up System (Promega, Beijing, China). The DNA samples were sequenced using

Send correspondence to Zhen Wei. Zhengzhou University, School of Life Sciences, Kexue Avenue 100, 450000, Zhengzhou, Henan, China. E-mail: weizhen@zzu.edu.cn.

\footnotetext{
* These authors contributed equally to this work.
}

Illumina HiSeq Xten Platforms. Cp genomes were assembled using NOVOPlasty v4.0 (Dierckxsens et al., 2016), annotated and manually corrected in Geneious Prime 2020.1.2 (Kearse et al., 2012). The structures and genes of the cp genomes were visualized by OrganellarGenomeDRAW (Lohse et al., 2013). All sequenced cp genomes were submitted to GenBank (Table S2).

All Swertia cp genomes have typical cp genome structures, including a large single copy region (LSC), a small single copy region (SSC), and two inverted repeat regions (IRs). A gene map of Swertia bimaculata Hook.f. \& Thomson ex C.B. Clarke (MW344293) was shown as an example (Figure 1), and other new cp genomes are shown in supplementary figures (Figure S1-S9). The length, GC content, and gene number of 15 Swertia species and seven relatives in Swertiinae were determined and compared (Table S3). The total length of Swertia cp genomes range from 149,488 bp to $154,097 \mathrm{bp}$. The total GC content of the cp genomes are relatively stable $(38.0 \%-38.2 \%)$, while the GC content of the IRs are higher than that of single copy regions. The cp genomes of other related species did not show any distinct differences from Swertia species. Most Swertia cp genomes contain 134 genes, including 85 protein coding genes, eight rRNA genes, 37 tRNA genes, and four pseudogenes (rps 16, infA, $y c f 1$ and rps19 genes) (Table S4). Gene deletion and additional pseudogenes were found in some Swertia cp genomes, as results of additional termination codons, complete or partial gene loss (Table S5). Previous studies have shown 


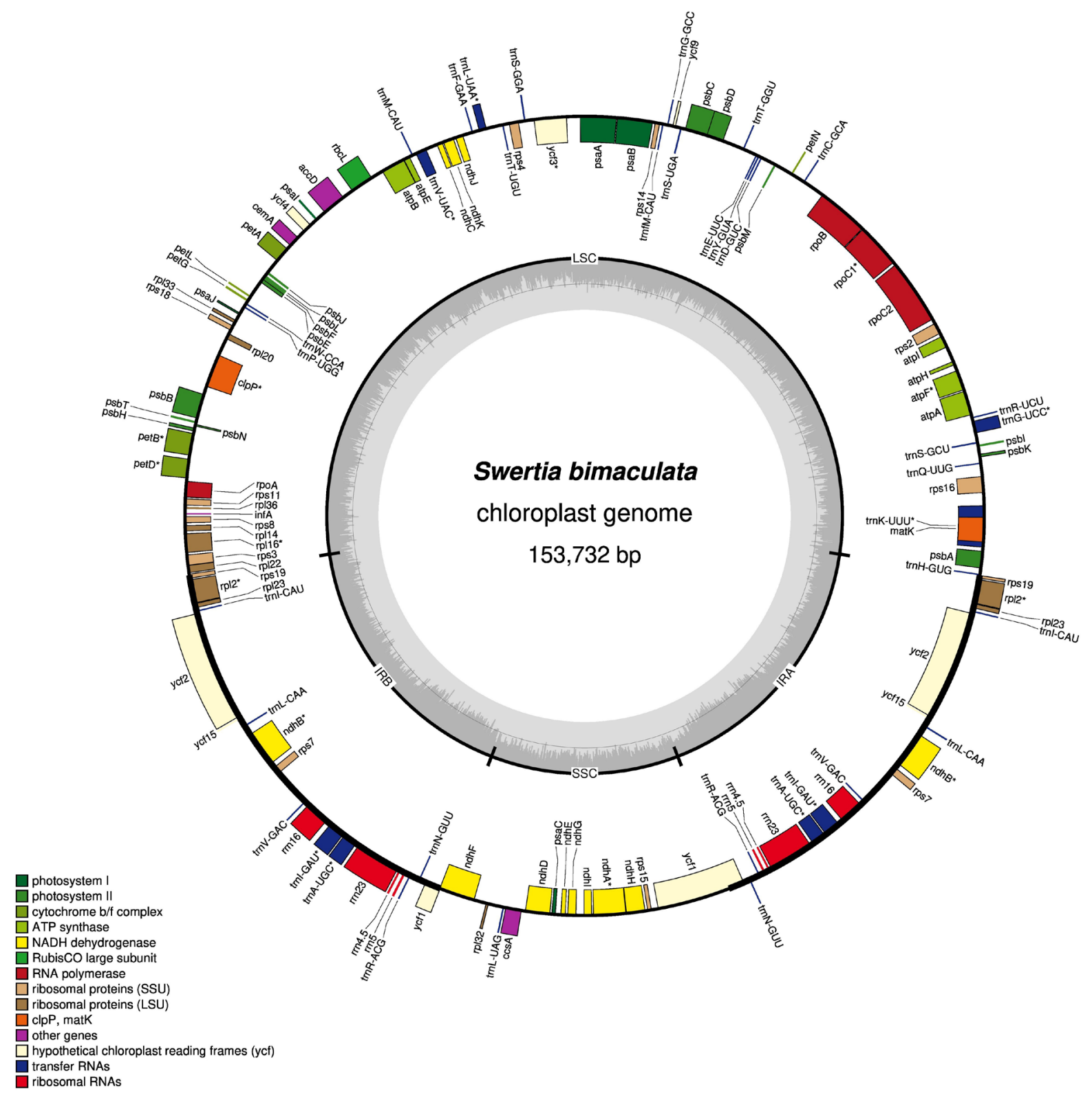

Figure 1 - Gene map of the Swertia bimaculata (MW344293) chloroplast genome. The translation of genes outside the outer circle occurs in a counterclockwise direction, while the translation of genes inside occurs in a clockwise direction. The dark and light gray colors in the inner circle represent GC and AT content, respectively. Different functional gene groups are highlighted by different colors.

that some genes with defects or loss in cp genomes may have other copies with normal function in nuclear genomes, which could help avoid death or damage during plant growth (Martin et al., 1998; Sugiura et al., 2003).

The mVISTA software was used to evaluate the sequence conservations among genomes (https://genome.lbl.gov/vista/ index.shtml). The structures and sequences of Swertia cp genomes are conserved, especially in the IR regions (Figure S10), probably due to the existence of rRNA genes and gene conversion (Khakhlova and Bock, 2006). Variation rates of coding and noncoding regions were calculated using Geneious Prime 2020.1.2. The results showed the variation rates of coding regions are generally lower than those of noncoding regions (Figure 2), and the variation rates of noncoding regions in the IR are lower than those in other regions. These regions of high variations could be used to develop new DNA barcodes of Swertia species and two pairs of newly designed primers were provided in the supplements (Table S6). Differences in the four boundaries of the LSC, SSC, and IRs were illustrated using the IRscope online website (https://irscope.shinyapps. io/irapp/). Boundary comparisons in Swertia species found that the structures of boundaries are conservative with slight differences among taxa (Figure 3). The boundaries of the four regions between Swertia and its relatives showed expansion and contraction to some extent.

Phylogenetic trees were constructed based on 54 complete cp genomes of Gentianeae species and an Apocynaceae species as outgroup. Additional $\mathrm{cp}$ genomes used in the phylogenetic analysis were downloaded from the National Center for Biotechnology Information database (https:// www.ncbi.nlm.nih.gov/) (Table S2). We applied Bayesian inference (BI), maximum likelihood (ML), and maximum 

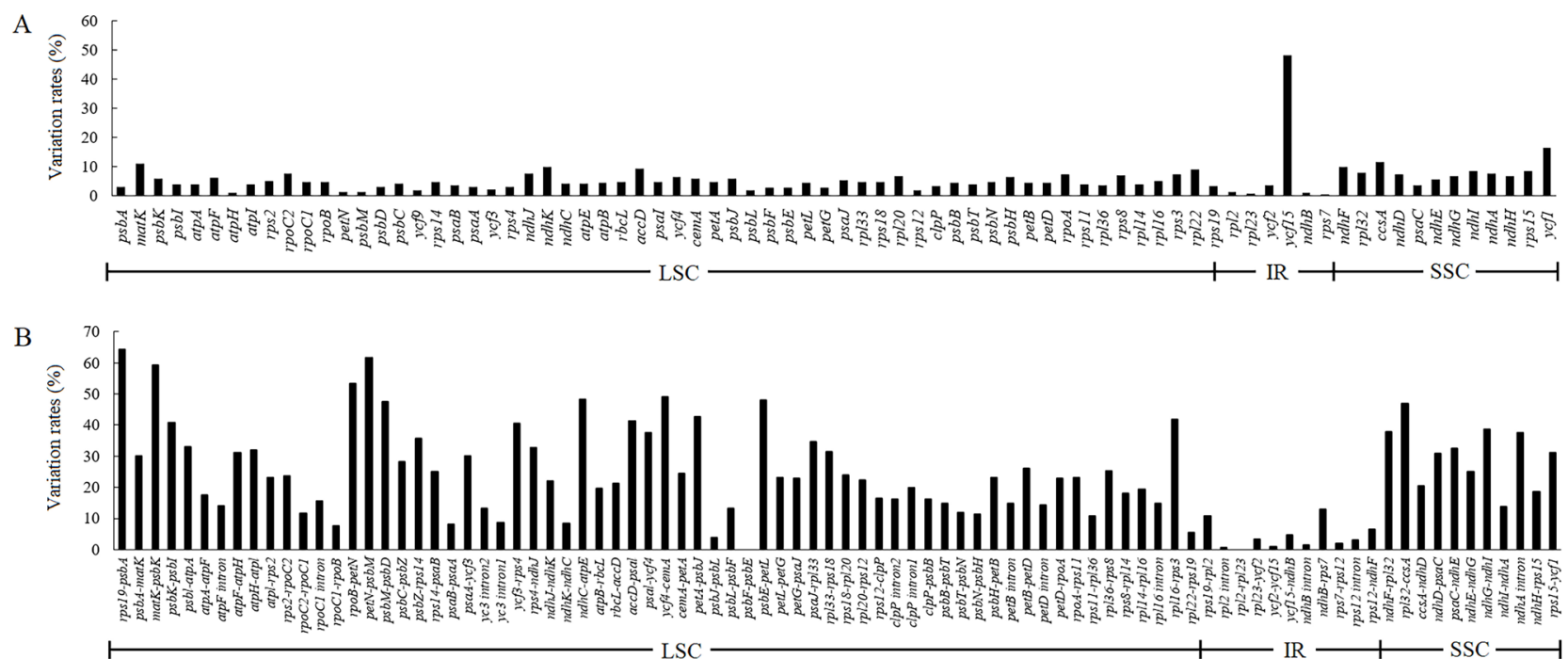

Figure 2 - Variation rates of coding and noncoding regions in the chloroplast genomes of 15 Swertia species. A: Variation rates of 78 protein coding genes; the $\mathrm{y}$-axis represents protein coding genes. B: Variation rates of noncoding regions; the $\mathrm{y}$-axis represents intergenic spacers or introns. Abbreviations: LSC- large single copy region; SSC-small single copy region; IR- inverted repeat region.

parsimony (MP) methods to construct phylogenetic trees. The ML tree and BI tree were constructed in CIPRES Science Gateway (https://www.phylo.org/portal2/login!input.action) using RAxML-HPC2 on XSEDE and MrBayes on XSEDE. For ML tree, GTR+GAMMA was chosen as the model for the bootstrapping phase. The bootstrap replicates were 5000, and "print branch lengths (-k)" was chosen under "Configure Bootstrapping". Other parameters were default settings. For $\mathrm{BI}$ analyses, the parameters were: 1 set $\mathrm{nst}=6$ rates $=$ gamma; unlink statefreq $=($ all $)$ revmat $=($ all $)$ shape $=($ all $)$ pinvar $=($ all $)$; prset applyto $=($ all $)$ ratepr $=$ variable; mcmcp ngen $=5000000$, relburnin $=$ yes, burninfrac $=0.25$, printfreq $=$ 1000 , samplefreq $=5000$, nchains $=4$ temp $=0.05$. Other parameters were default settings. The MP tree was structured using PAUP v4.0a with bootstrap 3000 and default parameters. The output tree files were modified in TreeGraph 2 (http:// treegraph.bioinfweb.info/) and Figtree v1.4.3 (http://tree.bio. ed.ac.uk/software/figtree/).

Phylogenetic trees based on three methods had the same topologies (Figure 4). All Gentianeae species formed two monophyletic clades: Gentianinae and Swertiinae. Swertia formed a large clade with Comastoma Toyokuni, Halenia Borkh., Lomatogoniopsis T.N. Ho \& S.W. Liu, Lomatogonium A. Braun and Veratrilla Franch, indicating that Swertia is a complex polyphyletic group. Swertia, Comastoma, Halenia, Lomatogoniopsis, Lomatogonium and Veratrilla were traditionally thought to be independent taxonomic units based on morphology (Ho and James, 1995), though many of the species were used as 'Zangyinchen' or 'Dida' medicines to treat hepatobiliary diseases. Similar results of polyphyletic relationships were reported previously in studies of Swertiinae species based on fragments of $\mathrm{cp}$ and nuclear genomes. One possible explanation for such discrepancies is that morphological features are plastic (Kadereit and von Hagen, 2003); they may be affected by many factors, such as heredity and environment, and character changes may not always be revealed by molecular data. Additionally, S. hispidicalyx and $S$. dilatata are sister groups and their rpl33 genes were completely lost in the cp genomes. This gene loss might have contributed to their close relationships in the phylogenetic tree. To maintain the stability of concept, Swertia species should be divided into smaller genera or merged with Comastoma, Halenia, Lomatogoniopsis, Lomatogonium and Veratrilla into larger genera. A combination of morphological data and nuclear genome data will be required in future research to resolve the phylogenetic relationships among genera in Swertiinae.

Selection pressure was analyzed in Datamonkey (http:// www.datamonkey.org/) using the Fixed Effects Likelihood Method. The values of non-synonymous/synonymous rate ratios $(\mathrm{dn} / \mathrm{ds})$ were calculated based on the 78 protein coding genes of 15 Swertia species, ranging from 0 to 1.65 (Figure $\mathrm{S} 11)$. The ratios of petL (1.34) and psaI (1.65) were greater than one, meaning the two genes are under the influence of positive selection. Most genes with dn/ds values between zero and one are under purifying selection. A few genes have $\mathrm{dn} / \mathrm{ds}$ values close to one, including $a t p F, c e m A, p s b N, y c f 2, y c f 15$, and $y c f 1$ genes. These genes may be subject to weak positive selection. The Swertia plants studied here are mainly distributed across the Qinghai-Tibet plateau and other high-altitude areas, which are subject to large temperature differences between day and night, low overall temperatures, and high levels of ultraviolet radiation (Körner, 2003). When non-synonymous mutations can provide more survival opportunities for species, their rates can exceed that of synonymous mutations over a long period and be preserved in the population (Wolfe and ó'hUigín, 2016). The psaI gene codes for the psaI subunit of photosystem I (PSI) in plants and plays a role in the trimerization of PSI by stabilizing the combination of $p s a L$ to the light-harvesting complex (Plöchinger et al., 2016). The petL gene codes for subunits of the cytochrome $b 6 f$ complex, involved in electron transport from photosystem II (PSII) to PSI (Schwenkert et al., 2007). The positive selection on $p s a I$ and pet $L$ genes may reflect the adaptation of Swertia to the light environment of the Qinghai-Tibet plateau. 


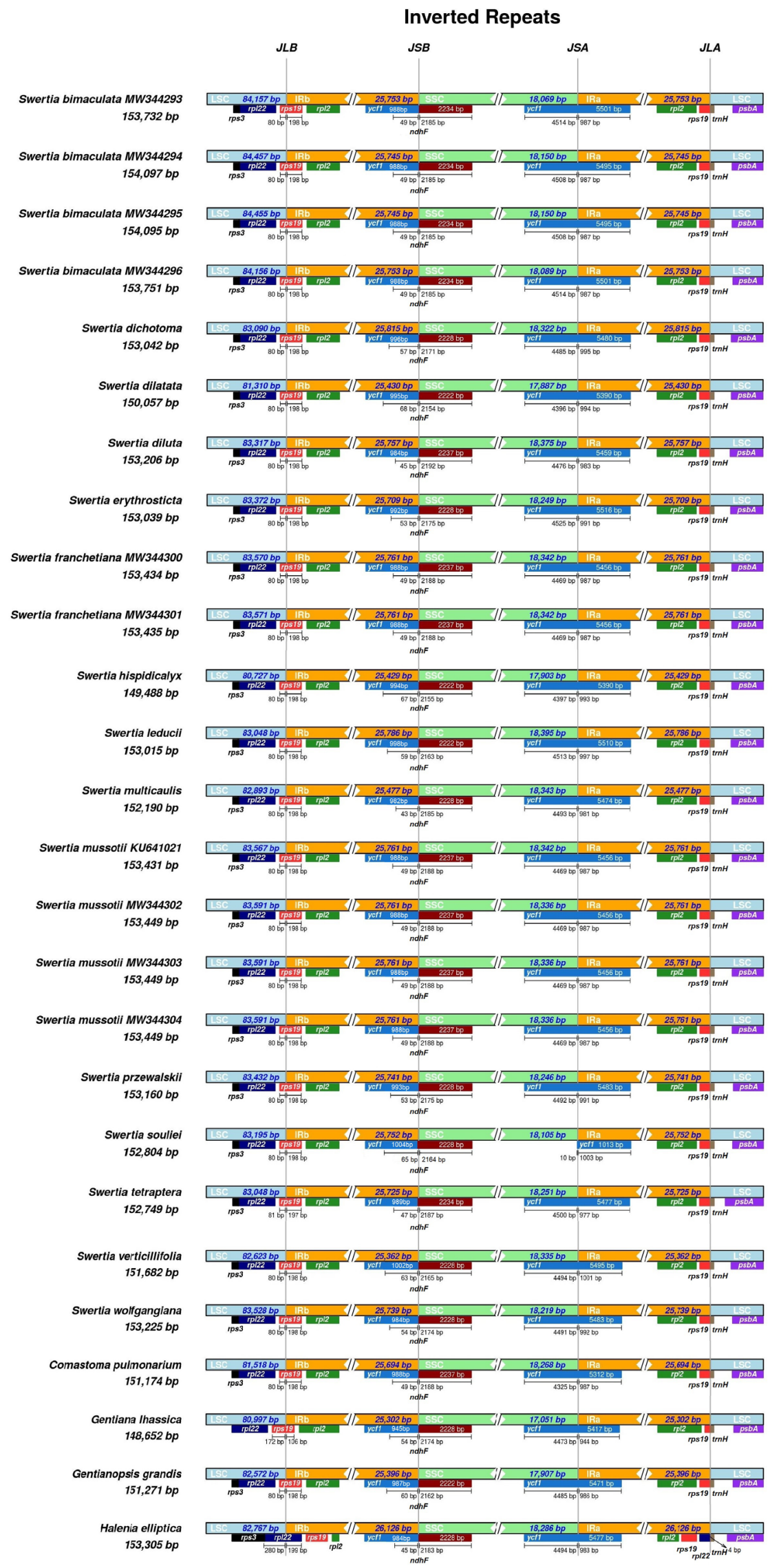

Figure 3 - Boundaries of the LSC, SSC, and IRs in chloroplast genomes of 15 Swertia and four related species. LSC: large single copy region; SSC: small single copy region; IRa and IRb: two inverted repeat regions; JLB: junction between the LSC and IRb; JSB: junction between the SSC and IRb; JSA: junction between the SSC and IRa; JLA: junction between the LSC and IRa. 


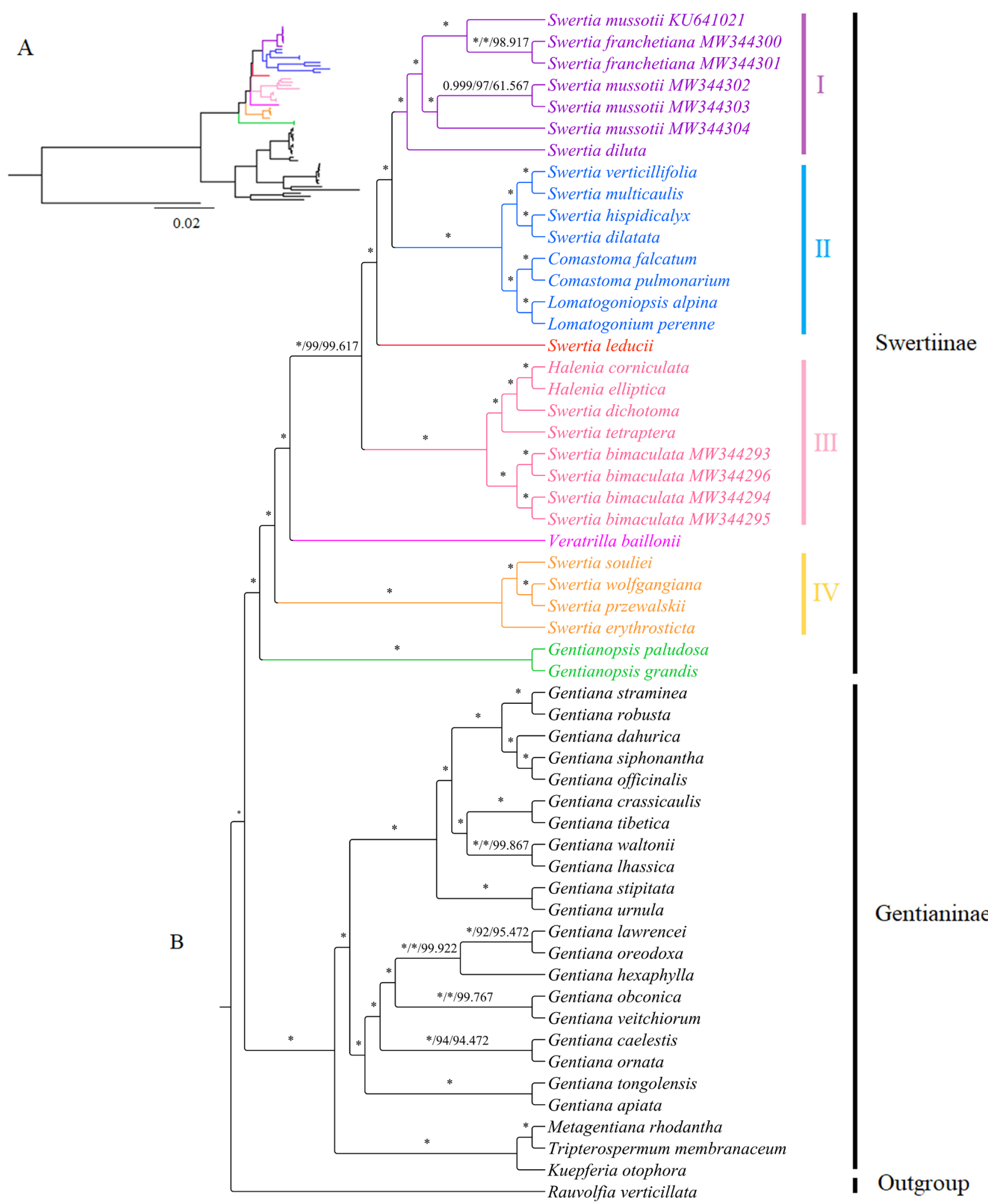

Figure 4 - Phylogenetic tree of 54 Gentianeae species and one outgroup based on the complete chloroplast genomes. A: tree with branch lengths; B: tree without branch lengths. Supporting values are shown in the form of BI/ML/MP. The symbol * indicates full support. Abbreviations: BI: Bayesian inference; ML: maximum likelihood; MP: maximum parsimony. 
Overall, we described here 16 newly sequenced cp genomes (10 species) of Swertia, and made comparative, phylogenetic and selection pressure analyses of them and other related species. Our results indicated that Qinghai Swertia species form a polyphyletic group and have similar structures and characteristics in cp genomes. Two genes were clearly under positive selection in Swertia cp genomes ( $p s a I$ and pet $L$ genes), while some other genes were likely subject to weak selection. The results in this study could be utilized for developing DNA barcodes for medicinal Swertia plants and further phylogenetic study in Swertia and Swertiinae.

\section{Acknowledgements}

This work was supported by the National Natural Science Foundation of China (31700175 to Z.W.; 31670188 to S.Z.); the Natural Science Foundation of Qinghai Department of Science and Technology (2019-ZJ-925 to J.L.).

\section{Conflict of Interest}

The authors declare that they have no conflict of interest.

\section{Author Contributions}

ZW and JL, conceived and the study; XX, RC, ML, HW, KS and SW conducted the experiments; XX, RC, ML, KS, YS and SW analyzed the data; XX, ZW and JL wrote the manuscript; YS, SZ, JL and ZW, reviewed and edited the manuscript, supervised the students; HW, JL and SZ, field investigation and material collection. All authors have read and approved the final version.

\section{References}

Dierckxsens N, Mardulyn P and Smits G (2016) NOVOPlasty: de novo assembly of organelle genomes from whole genome data. Nucleic Acids Res 45:e18.

Ho TN and James SP (1995) Flora of China. 1st edition. Missouri Botanical Garden Press, Saint Louis.

Ho TN, Xue CY and Wang W (1994) The origin, dispersal and formation of the distribution pattern of Swertia L. (Gentianaceae). Acta Phytotaxon Sin 32:525-537.

Joshi K and Joshi A (2008) Swertia L. (Gentianaceae) in Nepal Himalaya: Checklist, phytogeography, ethnobotany and conservation status. Ethnobot Leafl 12:361-372.

Kadereit JW and von Hagen KB (2003) The evolution of flower morphology in Gentianaceae-Swertiinae and the roles of key innovations and niche width for the diversification of Gentianella and Halenia in South America. Int J Plant Sci 164:S441-S452.

Kearse M, Moir R, Wilson A, Stones-Havas S, Cheung M, Sturrock S, Buxton S, Cooper A, Markowitz S, Duran C et al. (2012) Geneious Basic: An integrated and extendable desktop software platform for the organization and analysis of sequence data. Bioinformatics 28:1647-1649.

Khakhlova O and Bock R (2006) Elimination of deleterious mutations in plastid genomes by gene conversion. Plant J 46:85-94.

Körner C (2003) Alpine plant life: Functional plant ecology of high mountain ecosystems. 2nd edition. Springer, Berlin, 349 p.

Lohse M, Drechsel O and Bock R (2013) OrganellarGenomeDRAWa suite of tools for generating physical maps of plastid and mitochondrial genomes and visualizing expression data sets. Nucleic Acids Res 41:575-581.

Martin W, Stoebe B, Goremykin V, Hansmann S, Hasegawa M and Kowallik KV (1998) Gene transfer to the nucleus and the evolution of chloroplasts. Nature 393:162-165.
Plöchinger M, Torabi S, Rantala M, Tikkanen M, Suorsa M, Jensen PE, Aro EM and Meurer J (2016) The low molecular weight protein PsaI stabilizes the light-harvesting complex II docking site of Photosystem I. Plant Physiol 172:450-463.

Schwenkert S, Legen J, Takami T, Shikanai T, Herrmann RG and Meurer J (2007) Role of the low-molecular-weight subunits PetL, PetG, and PetN in assembly, stability, and dimerization of the cytochrome b6f complex in tobacco. Plant Physiol 144:1924-1935.

Sugiura C, Kobayashi Y, Aoki S, Sugita C and Sugita M (2003) Complete chloroplast DNA sequence of the moss Physcomitrella patens: Evidence for the loss and relocation of $r p o A$ from the chloroplast to the nucleus. Nucleic Acids Res 31:5324-5331.

von Hagen KB and Kadereit JW (2001) The phylogeny of Gentianella (Gentianaceae) and its colonization of the southern hemisphere as revealed by nuclear and chloroplast DNA sequence variation. Org Divers Evol 1:61-79.

von Hagen KB and Kadereit JW (2002) Phylogeny and flower evolution of the Swertiinae (Gentianaceae-Gentianeae): Homoplasy and the principle of variable proportions. Syst Bot 27:548-572.

Wei Z, Zhu SX, Berg RGVd, Bakker FT and Schranz ME (2017) Phylogenetic relationships within Lactuca L. (Asteraceae), including African species, based on chloroplast DNA sequence comparisons. Genet Resour Crop Evol 64:55-71.

Wolfe K and Ó'hUigín C (2016) Significance of positive selection and gene duplication in adaptive evolution: in memory of Austin L. Hughes. Immunogenetics 68:749-753.

Xi HC, Sun Y and Xue CY (2014) Molecular phylogeny of Swertiinae (Gentianaceae-Gentianeae) based on sequence data of ITS and matK. Plant Divers 36:145-156.

Zhong GY, Gu R, Zhou HR, Wang CH, Zhao JF, Liu X, Ze-Weng YZ and Qin SY (2009) Textual criticized and collated on names and breeds of Tibetan medicine "Dida". Zhongguo Zhong Yao Za Zhi 23:3139-3144.

\section{Supplementary material}

The following online material are available for this article:

Table S1 - Information of plant materials.

Table S2 -Accession numbers of the chloroplast genomes in this study. Table S3 - Characteristics of chloroplast genomes from 15 Swertia species and seven related species.

Table S4 - Genes in Swertia chloroplast genomes.

Table S5 - The pseudogenes in Swertia chloroplast genomes.

Table S6 - Information of primer sequences.

Figure S1 - Gene maps of $S$. dichotoma chloroplast genome.

Figure S2 - Gene maps of $S$. dilatata chloroplast genome.

Figure S3 - Gene maps of $S$. diluta chloroplast genome.

Figure S4 - Gene maps of S. erythrosticta chloroplast genome.

Figure S5 - Gene maps of S. franchetiana chloroplast genome.

Figure S6 - Gene maps of S. mussotii chloroplast genome.

Figure S7 - Gene maps of S. przewalskii chloroplast genome.

Figure S8 - Gene maps of S. tetraptera chloroplast genome.

Figure S9 - Gene maps of $S$. wolfgangiana chloroplast genome.

Figure S10 - The alignment of chloroplast genomes for 15 Swertia species and four related species. $S$. wolfgangiana was used as a reference.

Figure S11 - Non-synonymous/synonymous rate ratios of the protein coding genes from the chloroplast genomes of 15 Swertia species.

Associate Editor: Rogério Margis

License information: This is an open-access article distributed under the terms of the Creative Commons Attribution License (type CC-BY), which permits unrestricted use, distribution and reproduction in any medium, provided the original article is properly cited. 